\title{
Erratum to: Synthetic and natural antioxidants attenuate cisplatin-induced vomiting
}

\author{
Javaid Alam, Fazal Subhan ${ }^{1 *}$, Ihsan Ullah², Muhammad Shahid', Gowhar Ali' and Robert D. E. Sewell ${ }^{3}$
}

\section{Erratum}

After publication of the original article [1], it was brought to our attention that the unit for concentration in the first column of Table 2 should read $\mu \mathrm{g} / \mathrm{mL}$. In addition, the $9^{\text {th }}$ row in Table 2 should read $\mathrm{EC}_{50}(\mu \mathrm{g} / \mathrm{mL})$.

The original article was corrected. The corrections have been published in this erratum for quick reference. We apologise for any confusion this may have caused.

\section{Author details}

'Department of Pharmacy, University of Peshawar, Peshawar 25120, Khyber Pakhtunkhwa, Pakistan. ${ }^{2}$ Department of Pharmacy, University of Swabi, Swabi, Pakistan. ${ }^{3}$ Cardiff School of Pharmacy and Pharmaceutical Sciences, Cardiff

University, Cardiff CF103NB, UK.

Received: 25 January 2017 Accepted: 25 January 2017

Published online: 01 February 2017

\section{Reference}

1. Alam J, Subhan F, Ullah I, Shahid M, Ali G, Sewell RDE. Synthetic and natural antioxidants attenuate cisplatin-induced vomiting. BMC pharmacol toxicol. 2017;18(4):1-11.

\footnotetext{
* Correspondence: fazal_subhan@upesh.edu.pk

'Department of Pharmacy, University of Peshawar, Peshawar 25120, Khyber Pakhtunkhwa, Pakistan
} 
Table 2 Percent of DPPH free radical scavenging activity and antioxidant strength of N-(2-mercaptopropionyl) glycine (MPG), vitamin C (Vit- C), grape seed proanthocyanidin (GP), B. monnieri n-butanolic fraction (BM-ButFr) or standard butylated hydroxytoluene $(\mathrm{BHT})$ against their respective concentrations

\begin{tabular}{|c|c|c|c|c|c|}
\hline \multirow{2}{*}{$\begin{array}{l}\text { Concentration } \\
(\mu \mathrm{g} / \mathrm{mL})\end{array}$} & \multicolumn{5}{|c|}{ Percent inhibition (\%) } \\
\hline & $\mathrm{BHT}$ & MPG & Vit-C & GP & BM-ButFr \\
\hline$\overline{1}$ & $15.0 \pm 0.7$ & $20.0 \pm 2.1$ & $14.6 \pm 1.5$ & $36.4 \pm 0.8$ & $24.5 \pm 0.7$ \\
\hline 10 & $24.4 \pm 4.2$ & $20.8 \pm 1.8$ & $15.7 \pm 0.6$ & $71.6 \pm 0.7$ & $20.9 \pm 0.8$ \\
\hline 30 & $22.1 \pm 1.4$ & $19.4 \pm 1.2$ & $17.3 \pm 1.5$ & $82.6 \pm 1.2$ & $32.2 \pm 2.4$ \\
\hline 50 & $22.8 \pm 0.5$ & $24.4 \pm 1.0$ & $21.2 \pm 2.4$ & $92.4 \pm 0.1$ & $46.5 \pm 2.9$ \\
\hline 100 & $58.6 \pm 3.8$ & $94.0 \pm 0.01$ & $92.7 \pm 0.7$ & $92.4 \pm 0.2$ & $90.9 \pm 0.5$ \\
\hline 200 & $89.7 \pm 2.4$ & $96.2 \pm 0.2$ & $96.0 \pm 0.3$ & $91.6 \pm 0.3$ & $90.7 \pm 0.3$ \\
\hline 500 & $93.8 \pm 0.1$ & $96.0 \pm 0.7$ & $96.7 \pm 0.2$ & $89.6 \pm 0.2$ & $89.9 \pm 0.4$ \\
\hline \multicolumn{6}{|l|}{ Antioxidant strength } \\
\hline $\mathrm{EC}_{50}(\mu \mathrm{g} / \mathrm{mL})$ & $98.17 \pm 3.842$ & $67.66 \pm 3.095$ & $69.42 \pm 3.027$ & $6.498 \pm 0.630$ & $55.61 \pm 1.137$ \\
\hline Antiradical power & $0.0102 \pm 0.0004$ & $0.0148 \pm 0.0007$ & $0.0143 \pm 0.0005$ & $0.1567 \pm 0.0145$ & $0.0159 \pm 0.0010$ \\
\hline Stoichiometry & $196.3 \pm 7.685$ & $135.3 \pm 6.191$ & $138.8 \pm 6.053$ & $13.00 \pm 1.261$ & $111.2 \pm 2.274$ \\
\hline
\end{tabular}

Values are expressed as mean \pm S.E.M from three separate experiments 